\title{
GPU-accelerated Monte Carlo based scatter correction in brain PET/MR
}

\author{
Michaela Gaens $^{1 *}$, Julien Bert ${ }^{2}$, Uwe Pietrzyk ${ }^{1,3}$, A Autret $^{2}$, N Jon Shah ${ }^{1}$, Dimitris Visvikis ${ }^{2}$ \\ From PSMR14: 3rd Conference in PET/MR and SPECT/MR \\ Kos Island, Greece. 19-21 May 2014
}

'Institute of Neuroscience and Medicine - 4, Forschungszentrum Juelich, Germany
To develop and evaluate a fast and accurate Monte Carlo simulation (MCS) based scatter correction for PET/MR imaging, based on Geant4 using graphical processing units (GPUs).

The GPU implementation by Bert et al. [1] including the photon tracking within a voxelized volume was extended with the modeling of the detection process in the Siemens MR-BrainPET. Using NVIDIA's CUDA photon pairs undergo several kernel functions in parallel, from their generation according to the activity distribution up to their detection in the scanner. Subsequently, coincidences are sorted and stored in list-mode. The new GPU implementation (GeForce GTX690) was validated by comparison with Gate v6.2 on a single CPU (Intel Core i7, 3.4GHz), showing a speedup factor of 135 . The use of multiple GPUs can further increase this acceleration.

For Compton scatter correction the reconstructed emission image, the patient MR-derived attenuation map and a CT-derived attenuation template of the MR coils are used as input for the simulation. The resulting simulated projection data are scaled to the normalized measured projection data. Thus, the often unstable fit to the distribution tails, necessary for single scatter simulation (SSS)[2], is circumvented. The scaled simulated scatter distribution is then incorporated in the reconstruction. The proposed scatter correction methodology was evaluated and compared to the SSS approach using sphere phantom measurements. Finally, the effect on patient data was investigated.

Phantom results suggest an improved contrast with our new MC-based scatter correction, especially for measurements including the MR coils, where the contrast recovery was improved by $10-14 \%$. First patient images also show improved grey/white matter contrast.

Table 1 Comparison of recovery coefficients after scatter correction based on MCS and SSS. MC-based scatter correction shows improved recovery coefficients for the hot spheres.

\begin{tabular}{lllllll}
\hline Recovery Coefficient [\%] & $27 \mathrm{~mm}$ cold & $22 \mathrm{~mm}$ cold & $17 \mathrm{~mm}$ hot & $13 \mathrm{~mm}$ hot & $10 \mathrm{~mm}$ hot & $8 \mathrm{~mm}$ hot \\
\hline MCS & 82.5 & 79.3 & 95.4 & 85.0 & 71.6 & 80.0 \\
\hline SSS & 81.8 & 77.8 & 80.6 & 72.1 & 60.5 & 65.9 \\
\hline Difference & 0.7 & 1.5 & 14.8 & 12.9 & 11.1 & 14.1 \\
\hline
\end{tabular}

(c) 2014 Gaens et al; licensee Springer This is an Open Access article distributed under the terms of the Creative Commons Attribution License (http://creativecommons.org/licenses/by/4.0), which permits unrestricted use, distribution, and reproduction in any medium, provided the original work is properly cited. 

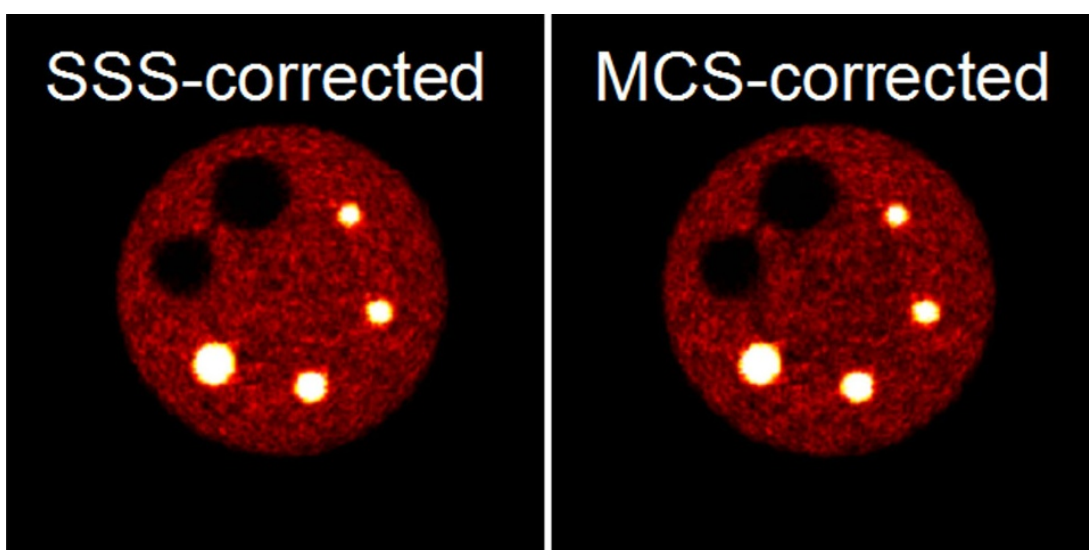

Figure 1 Assessment of image quality according to NEMA NU 2-2007. Images show the phantom with a hot spheres/background ratio of 8:1 measured with MR head coils inside the BrainPET scanner.

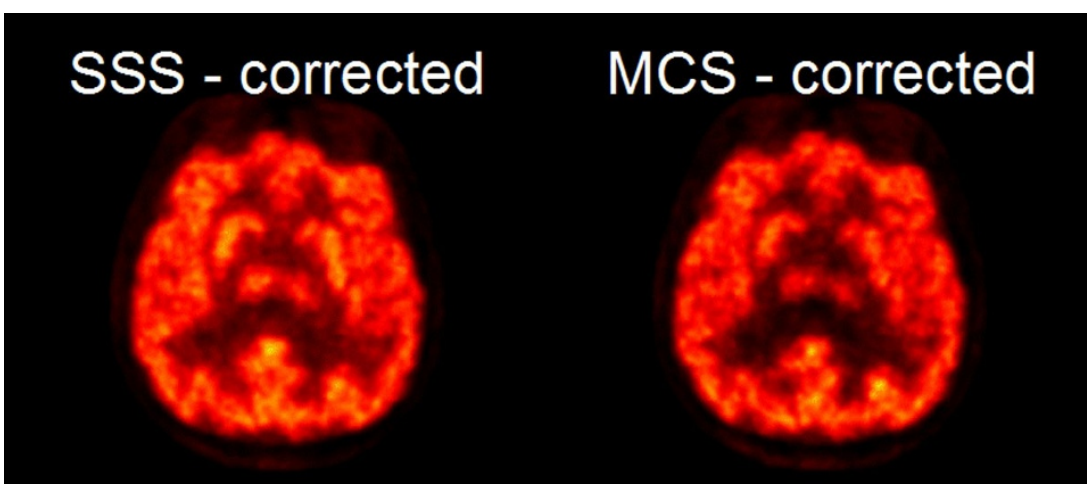

Figure 2 Comparison of reconstructed FDG images using the scatter estimate provided by the vendor's SSS (left) and our MCS method (right). Images were reconstructed using the vendor provided 3D OPOSEM algorithm with 2 subsets and 32 iterations.

The new GPU-accelerated MCS allows for fast and accurate estimation of scattered coincidences in PET studies using the Siemens MR-BrainPET with a major speed-up compared to standard MCS and superior image quality compared with the standard correction based on SSS.

\section{Authors' details}

${ }^{1}$ Institute of Neuroscience and Medicine - 4, Forschungszentrum Juelich, Germany. ${ }^{2}$ LaTIM, INSERM, UMR1101, CHRU Brest, France. ${ }^{3}$ Department of Mathematics and Natural Science, University of Wuppertal, Germany.

Published: 29 July 2014

\section{References}

1. Bert J, et al: Geant4-based Monte Carlo simulations on GPU for medical applications. Phys Med Biol 2013, 58:5593-5611.

2. Watson CC, Newport D, Casey ME: A single scatter simulation technique for scatter correction in 3D PET. ThreeDimensional Image Reconstruction in Radiology and Nuclear Medicine 1996, 4:255-268.

doi:10.1186/2197-7364-1-S1-A32

Cite this article as: Gaens et al:: GPU-accelerated Monte Carlo based scatter correction in brain PET/MR. EJNMMI Physics 2014 1(Suppl 1):A32. 